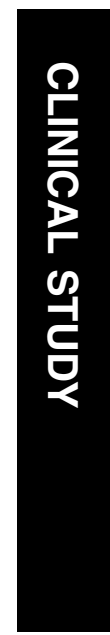

\title{
The efficacy and safety of $0.75 \%$ levobupivacaine vs $0.75 \%$ bupivacaine for peribulbar anaesthesia
}

\begin{abstract}
Background/aims Levobupivacaine, the $\mathrm{S}(-)$-enantiomer of racemic bupivacaine, is associated with a similar efficacy but a reduced potential for cardiovascular and central nervous system toxicity than racemic bupivacaine. Thus, this prospective, randomised, double-masked study was undertaken to assess the efficacy and safety of $0.75 \%$ levobupivacaine $v$ s $\mathbf{0 . 7 5 \%}$ bupivacaine, each with hyaluronidase, for peribulbar anaesthesia.
\end{abstract}

Methods A total of 60 patients undergoing elective anterior segment surgery were randomly allocated to receive either agent by a single, inferotemporal peribulbar injection technique, supplemented with a medial canthus injection if necessary. Ocular akinesia and orbicularis oculi function were assessed by scoring systems at 2 min intervals until satisfactory akinesia was achieved, and movements were reassessed on the day after surgery to confirm regression of the block. Results The time taken to reach a state of satisfactory anaesthesia and akinesia was deemed to be the primary measure of efficacy. Both agents achieved this in a similar median time of $2 \mathrm{~min}$ after receiving $5 \mathrm{ml}$ of the injectate, and the treatment difference was not statistically significant $(P=0.24)$. Blood

Department of Anaesthesia Derriford Hospital Plymouth, UK

\section{Correspondence:} Surgeon Commander DJ Birt

FRCA Royal Navy Principal Medical Officer HMS Illustrious BPFO 305, UK E-mail: 305@a.dii.mod.uk samples from the first 20 patients were taken at intervals up to $4 \mathrm{~h}$. These were analysed for plasma levels and confirmed similar plasma concentration $v$ s time profiles for the two agents. Seven patients in each group (23\%) complained of pain on injection but the technique was generally well tolerated. Two patients in the levobupivacaine group experienced serious adverse events, but neither was considered related to the study medication. The most common minor
DJ Birt and GC Cummings post-operative adverse event was prolongation of the local anaesthetic block, which was reported by nine patients (four in the levobupivacaine group and five in the bupivacaine group).

Conclusions Levobupivacaine and bupivacaine are equally successful in achieving clinically satisfactory peribulbar anaesthesia with few adverse effects. Eye (2003) 17, 200-206. doi:10.1038/ sj.eye. 6700313

Keywords: regional anaesthesia; peribulbar; bupivacaine; levobupivacaine

Introduction

Regional ocular anaesthesia was first performed by Köller in $1884^{1}$ using cocaine and represents the first description of a local anaesthetic technique. In the same year, Knapp ${ }^{2}$ instilled cocaine into the eye to produce a retrobulbar block, but a significant complication rate rat $^{3,4}$ restricted its popularity and there continued a need for a safer technique. Since the first description of the peribulbar block in 1986 by Davis, ${ }^{5}$ it has emerged as an extremely popular local anaesthetic technique for anterior segment surgery, particularly amongst nonopthalmologists. ${ }^{6-8}$

The most frequently used local anaesthetic mixtures include bupivacaine, as a racemic mixture, in combination with lidocaine and varying quantities of hyaluronidase. Although used extensively, racemic bupivacaine has occasionally displayed serious dose-related adverse drug reactions, and even death as a result of its cardiotoxicity. ${ }^{9,10}$ However, levobupivacaine, the isolated $\mathrm{S}(-)$-isomer of bupivacaine, has shown less cardiotoxicity than racemic 
bupivacaine in animal studies, both in vitro ${ }^{11}$ and in vivo, ${ }^{12}$ while possessing equivalent efficacy. ${ }^{13}$

The aim of this study was to compare the efficacy and safety of $0.75 \%$ levobupivacaine with a $0.75 \%$ racemic mixture of bupivacaine in peribulbar anaesthesia.

\section{Materials and methods}

With local ethics committee approval and written, informed consent, 60 day-case or overnight-stay patients undergoing elective anterior segment surgery, and aged 18 years or over, were recruited into the study over a 5-month period. Patients assessed as ASA IV, those with a severe visual handicap in the other eye, potentially pregnant women and those unable to give adequate consent were excluded.

The primary measure of efficacy was defined as the time taken to reach satisfactory akinesia for surgery (an akinesia score of 12 or more). It was felt that a betweengroup difference of $5 \mathrm{~min}$ for this variable would be clinically significant. A previous study estimated the between-patient standard deviation to be $6.2 \mathrm{~min}$ (KE Klubein and AP Rubin, personal communication with Inveresk Clinical Research). Therefore, a sample size of 30 patients in each group would provide a greater than $80 \%$ chance of detecting a 5 min difference $(\alpha=0.05) .{ }^{14}$

Patients were allocated randomly to one of two groups in blocks of 10 from a computer-generated series. Group 1 were to receive $0.75 \%$ levobupivacaine, and group 2 were to receive $0.75 \%$ bupivacaine, each with 75 units $/ \mathrm{ml}$ hyaluronidase. The trial drug was provided in patientspecific, sealed packaging. Initially, $10 \mathrm{ml}$ of the mixture was drawn into a syringe by a member of staff not otherwise involved in the study.

After recruitment, all patients were assessed preoperatively. Patient characteristics and details of concurrent disease and medication were recorded. No premedication was prescribed. An intravenous cannula was inserted into a superficial upper limb vein, and continuous ECG monitoring, noninvasive blood pressure measurement, and pulse oximetry were established.

With the patient in the supine position, 2-3 drops of $1 \%$ benoxinate were administered to provide conjunctival anaesthesia, and a pretreatment assessment of ocular movement was made in accordance with the scoring systems described below. After $2 \mathrm{~min}$, with the patient's eyes in the primary gaze position, a peribulbar block was performed by the same anaesthetist (GCC) in all subjects. A $31 \mathrm{~mm}, 25 \mathrm{G}$, long-bevelled needle was inserted inferotemporally through the skin, above the orbital rim, with the bevel towards the globe. The needle was advanced posteriorly beyond the equator of the globe, then given a slight superiomedial angulation towards the apex of the ocular muscle cone. At a depth of
Table 1 Orbicularis oculi scoring system

Assessor unable to manually force the eye open

Good closure but can be overcome manu Closure of the eye but is easily overcome by the assessor Eye just closing

Patient unable to close the eye

Table 2 General akinesia scoring system

Full movement 0

Almost full movement

Partial movement

Almost no movement

No movement

20-25 mm, and after aspiration, $5 \mathrm{ml}$ of the randomly assigned study drug was injected over 30-40 s.

The completion of this injection was deemed to be time zero for the purpose of subsequent assessments.

Following the injection, a Buys' mercury bag was placed over the closed and padded eyelid.

Akinesia of the four recti muscles, superior oblique, levator palpebrae superioris, and orbicularis oculi was assessed at 2, 4, 6, 8, 10, 15, 20, 25, and 30 min after the end of the first injection, or until a satisfactory block was achieved. Orbicularis oculi function was scored according to the scale shown in Table 1, and the remaining muscles were scored according to the scale shown in Table 2. Complete akinesia of all muscles would score 24 . An adequate block requiring no further injection of local anaesthetic was attained when the total score for the recti muscles superior oblique and levator palpebrae superioris had reached 12 and the orbicularis oculi score had reached 2 .

If after 10 min the block remained inadequate, a second injection could be administered medially between the caruncle and the medial canthus. A $25 \mathrm{~mm}, 25 \mathrm{G}$, longbevelled needle was directed posteriorly to a maximum of $15 \mathrm{~mm}$ and, after aspiration, a further $5 \mathrm{ml}$ of randomly assigned study drug was injected from the original syringe over $30-40 \mathrm{~s}$. The protocol also permitted a third injection of $3-5 \mathrm{ml}$ if the block remained inadequate after $15 \mathrm{~min}$.

The total volume of anaesthetic agent required was recorded along with an assessment of the degree of discomfort on injection (no pain $=0$, some pain $=1$, much pain $=2$ ). The quality of the operating conditions was assessed as excellent, satisfactory, or poor by the surgeon.

Pharmacokinetic blood samples were taken from the first 20 patients. A 16G intravenous cannula was sited, under $1 \%$ lidocaine, in the antecubital fossa and $6 \mathrm{ml}$ venous blood samples were taken before drug administration, at time 0 , and at $10,15,30,45,60,120$ 
and, where possible, $240 \mathrm{~min}$ after the placement of the block. These were centrifuged at $500 \mathrm{~g}$ for $10 \mathrm{~min}$ at $4^{\circ} \mathrm{C}$, and the plasma fraction assayed for drug concentration. Comparisons were made between the mean peak plasma drug concentrations $\left(C_{\max }\right)$ and the mean area under the plasma drug concentration vs time curves (AUC0- $t$ hrs where $t$ was the time of the last postinjection sample) for total bupivacaine and levobupivacaine. Levobupivacaine values were adjusted for dose formulation and divided by two for comparison with the levobupivacaine fraction of the racemic mixture.

At the routine ophthalmic review on the day after surgery, a further assessment of the eye movement was made in all patients to confirm regression of the block. Reports of mild, moderate or severe postoperative pain and any postoperative analgesia requirements were recorded by means of prescription charts or, in the case of day-case patients, from diary cards. Finally, 7 days after the operation, each patient was contacted by telephone to confirm their well-being.

The statistical package SAS (v6.07) was used to perform all statistical analyses on the clinical data. Since the akinesia assessments were made at set times and the time to satisfactory block could only take a limited number of values, an assumption of data normality would have been doubtful. The time to onset of a satisfactory block was, therefore, compared using a LOGIT model. Data relating to the degree of pain on injection, the degree of postoperative pain, and the quality of the surgical conditions were analysed using a LOGIT model or a Fisher's exact test as appropriate. The time to the first requirement for postoperative analgesia was compared using the Wilcoxon test. The plasma concentration data were analysed, assuming a noncompartment model, using the SIPHAR (v4.0) pharmacokinetic modelling package. All tests used a significance level of $5 \%$.

\section{Results}

A total of 60 patients were recruited, all of whom completed the study. Their demographic characteristics are shown in Table 3 and are similar for both groups.

All patients reported concurrent disease at preoperative screening, and with the exception of the endocrine, nutritional, metabolic, or immunity category,

Table 3 Patient characteristics

\begin{tabular}{lcc}
\hline & $0.75 \%$ levobupivacaine & $0.75 \%$ bupivacaine \\
\hline$n$ & 30 & 30 \\
F:M ratio & $2: 1$ & $2: 1$ \\
Mean age (range) (SD) & $76.6(60-90)(8.1)$ & $77.6(56-90)(8.7)$ \\
\hline
\end{tabular}

the occurrence of the various conditions was spread evenly between the two groups.

\section{Efficacy evaluation}

The primary efficacy variable was the time to onset of a block adequate for surgery. The results are summarised in Table 4, and illustrated in Figures 1 and 2. Statistical analysis produced an odds ratio of 0.51 , suggesting that the odds of levobupivacaine taking longer to achieve an adequate peribulbar block were almost twice that of bupivacaine. Despite this, the difference was not statistically significant at this sample size $(P=0.24)$.

Operative conditions were generally very good and there was no significant difference between the two groups in their quality $(P=0.52)$. However, two patients in the levobupivacaine group (who were operated on by the same surgeon) required supplementary injections to block orbicularis oculi.

Seven patients in each group complained of some pain on injection, but only one patient (in the bupivacaine group) complained of some pain postoperatively, and no patients complained of much pain. This result was

Table 4 Time to satisfactory block (min)

\begin{tabular}{lcc}
\hline & $0.75 \%$ levobupivacaine & 0.75\% \\
\hline Mean & 2.8 & 2.5 \\
SD & 1.1 & 0.9 \\
Median & 2.0 & 2.0 \\
Minimum & 2 & 2 \\
Maximum & 6 & 4 \\
$n$ & 30 & 30 \\
\hline
\end{tabular}

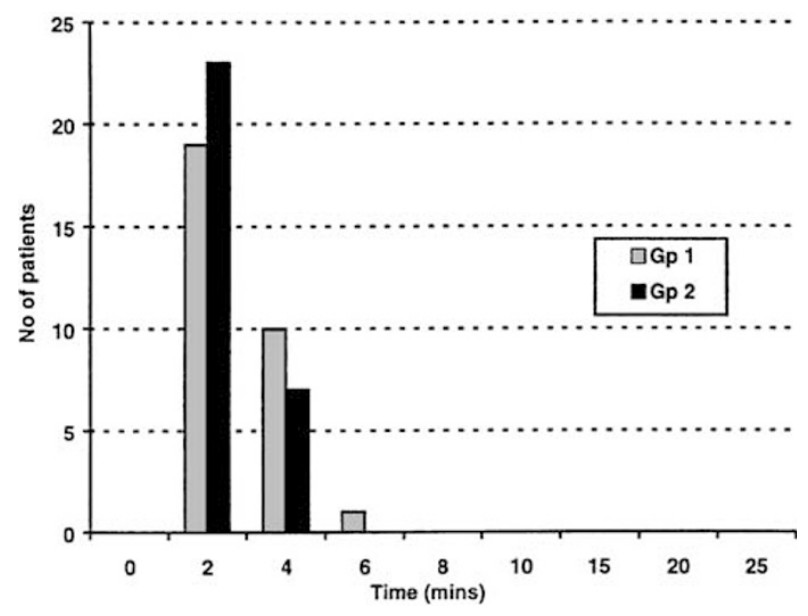

Figure 1 Distribution of the time taken to reach satisfactory block for the levobupivacaine group (Gp1) and the bupivacaine group (Gp2). 


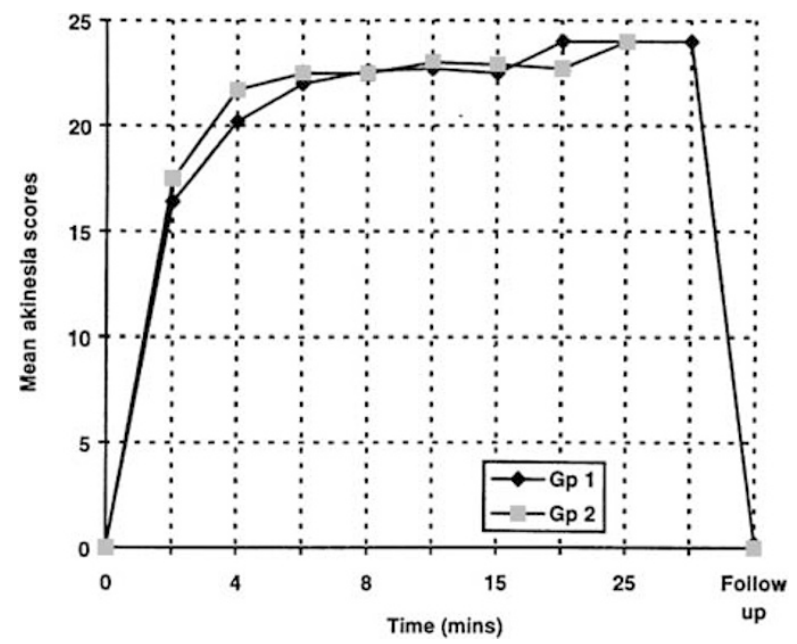

Figure 2 Mean ocular movement scores over time in the levobupivacaine (Gp1) and the bupivacaine (Gp2) groups.

shown to be nonsignificant $(P=1.00)$ using a Fisher's exact test. No significant differences were found between the groups in the time to first postoperative analgesia $(P=0.63)$.

\section{Safety evaluation}

A total of 25 adverse events (14 in the levobupivacaine group and 11 in the bupivacaine group) were reported by 22 patients and their distribution is summarised in Table 5.

Two patients (both in the levobupivacaine group) experienced serious adverse events during the study period. One patient (004) developed a postoperative urinary tract infection and the other (028) developed a subconjunctival haemorrhage during the surgical procedure. Both events involved an extension in the hospital stay and were, therefore, defined as serious. However, neither adverse event was judged to be related to the study medication.

Less serious adverse events that were judged to be possibly related to the study medication were reported by 16 patients (nine patients in the levobupivacaine group and seven in the bupivacaine group). The most commonly reported event (four patients in the levobupivacaine group and five in the bupivacaine group) was a prolongation of the local anaesthetic block confirmed by residual akinesia and diplopia on the day after surgery. This was judged as definitely related to the study medication. Eye pain occurring at some time during the first postoperative day was reported by four patients in the levobupivacaine group and three patients in the bupivacaine group. This was associated with diplopia, but both symptoms had invariably resolved by
Table 5 Adverse events

\begin{tabular}{lcc}
\hline & $\begin{array}{c}0.75 \% \\
\text { levobupivacaine }\end{array}$ & $\begin{array}{c}0.75 \% \\
\text { bupivacaine }\end{array}$ \\
\hline $\begin{array}{l}\text { Patients with adverse events } \\
\text { Patients with drug-related }\end{array}$ & 12 & 10 \\
adverse events & 8 & 7 \\
$\begin{array}{l}\text { Patients with serious adverse } \\
\text { events }\end{array}$ & 2 & 0 \\
$\begin{array}{l}\text { Deaths } \\
\text { arug-related adverse events are defined as those assessed by the } \\
\text { investigator as being possibly related or definitely related to the study } \\
\text { medication. }\end{array}$
\end{tabular}

the time of the initial postoperative review. These events were considered as possibly related to the study medications. All patients recovered fully, and the safety profiles of the two drugs were similar.

\section{Pharmacokinetic evaluation}

Mean plasma concentration $v s$ time profiles are illustrated on linear scales in Figures 3 and 4, and allowed the calculation of some pharmacokinetic values. Although there were no adverse clinical signs and the patient received adequate anaesthesia for surgery, data from patient 018 (levobupivacaine group) were excluded from the calculation as the concentration profile suggested that at least part of the injection may have been intravenous.

There was no significant difference between the mean values of the AUC0-t hrs and the $C_{\max }$ for the total bupivacaine profiles ( $P=0.38$ and 0.16 , respectively) or the adjusted levobupivacaine profiles $(P=0.78$ and 0.49 , respectively).

\section{Discussion}

This study has demonstrated that levobupivacaine and bupivacaine are equally successful in achieving clinically satisfactory peribulbar anaesthesia with few adverse effects.

The peribulbar technique we used was chosen owing to its safe reputation, being distant from the vessel-rich medial compartment, and the familiarity of this method to the authors. Although a $31 \mathrm{~mm}$ needle was preferred by the anaesthetist performing the blocks (GCC), the description of this unorthodox technique in this paper does not imply that it should be widely adopted. The slight superiomedial angulation delivers local anaesthesia deep into the peribulbar space and may partially account for the high success rate after a single injection when compared with previous studies. ${ }^{6,7}$ There was also a notably short onset time, which could have 


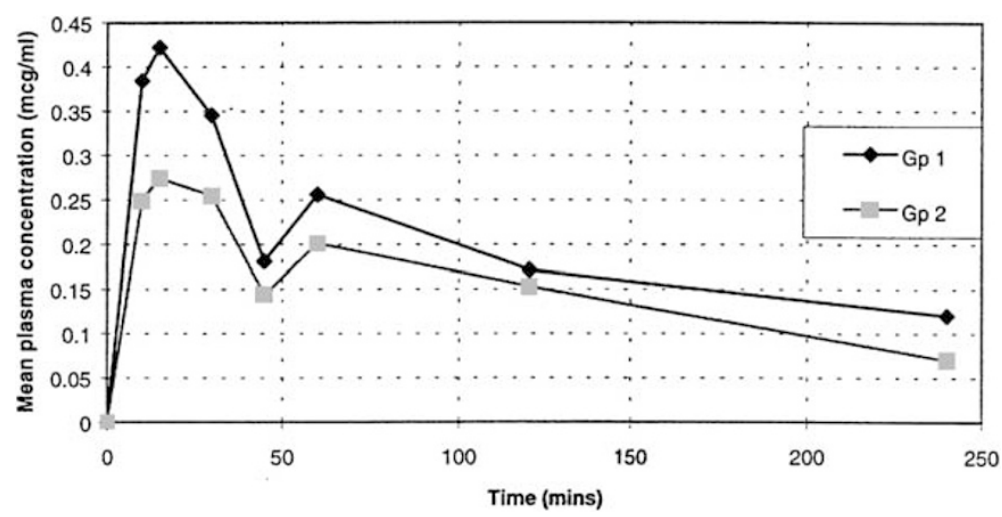

Figure 3 Plasma concentration values of total bupivacaine vs time for the levobupivacaine (Gp1) and bupivacaine (Gp2) groups.

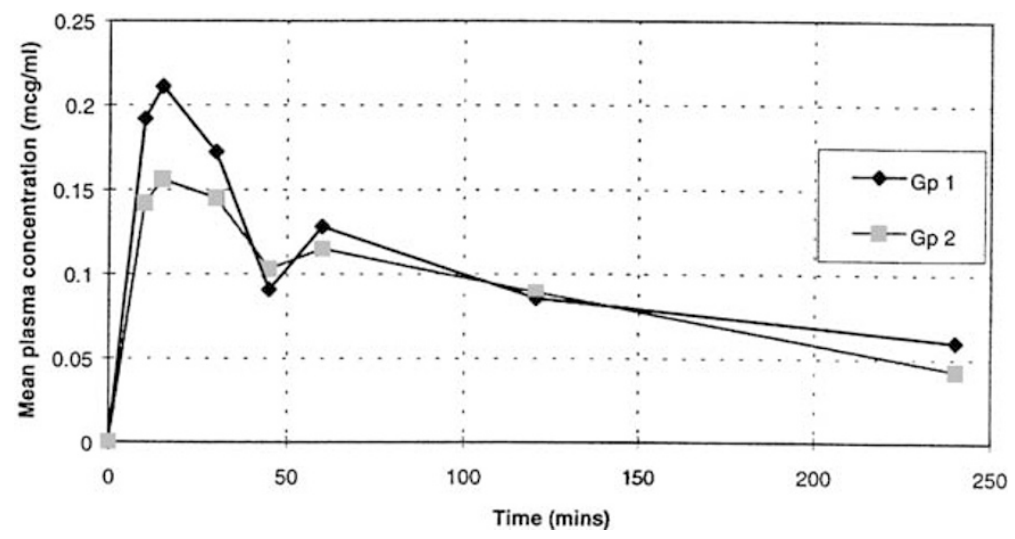

Figure 4 Plasma concentration values of levobupivacaine vs time for the levobupivacaine (Gp1) and bupivacaine (Gp2) groups.

been a result of the higher concentration of local anaesthetic used; with only one exception, an adequate block was achieved in either 2 or $4 \mathrm{~min}$. However, we did not make a direct comparison with the bupivacaine and lidocaine mixes used in most of these studies, and a separate study would be required to directly compare onset times and drug volumes.

Scoring systems are frequently used in the assessment of ocular muscle function. ${ }^{5,15-17}$ However, there is no accepted standard system - a fact that hinders comparison between studies. Our system resembled several other previously described methods, but any method using the sum of a scoring system as an end point can be criticised for its subjective influences, and the ability to achieve the same score through different observations. All our peribulbar blocks clearly achieved satisfactory scores in terms of the protocol, although two patients required supplementary injections of lidocaine to achieve full akinesia of the orbicularis oculi.

Nevertheless, we were satisfied that our scoring system and primary measure of efficacy were sufficiently sensitive to establish anaesthetic efficacy.
Both local anaesthetic preparations appeared to be well tolerated and produced good surgical conditions with few adverse reactions. What was disappointing was the relatively high number of patients ( $23 \%$ in each group) who complained of some pain during, or immediately after, the peribulbar injection. This may be attributable to the high concentration of the test drug, the relatively deep placement of the injection, or to the use of the transcutaneous route. The occurrence of prolonged blockade was a feature not previously reported, and is presumably related to the relatively high concentration of both the local anaesthetic preparations. Again, a direct comparison with bupivacaine/lidocaine mixtures may help to establish this connection.

Although bupivacaine remains an extremely popular anaesthetic, its use has been overshadowed by its toxicity profile. $\mathrm{LD}_{50}$ studies in rats and mice indicate the dextrobupivacaine isomer to be $30-40 \%$ more toxic than its levo-rotated counterpart. ${ }^{18}$ The predominant cause of death is a specific cardiotoxicity without antecedent hypoxia, resulting in arrhythmias and cardiovascular collapse. This was first reported in humans by Albright ${ }^{9}$ 
in 1979 as a series of six cases. The estimated total bupivacaine plasma level at which this occurs is $4-5 \mu \mathrm{g} / \mathrm{ml} .^{10}$ The plasma concentration from the racemic bupivacaine group demonstrated a mean peak plasma level of $0.281 \mu \mathrm{g} / \mathrm{ml}$, further supporting the safe reputation of the peribulbar technique.

The main mechanism for the cardiotoxicity is probably through its local anaesthetic action of blocking sodium channels. In animal preparations, this blockade results in a reduction of the maximum rate of depolarisation $\left(v\right.$-max) and a slowing of action potential conduction. ${ }^{19}$ This predisposes the myocardium to re-entrant arrythmias. Further animal studies comparing the isomers of bupivacaine indicate a higher affinity and, therefore, a greater inhibition of the $v$-max with dextrobupivacaine than with levobupivacaine. ${ }^{20}$ The dextro-enantiomer's greater affinity for myocardial potassium channels is even more marked, ${ }^{21}$ and this may also be partially responsible for its cardiotoxicity. Furthermore, there is evidence that the local anaesthetic action on the cardiovascular centre of the brain may make an indirect contribution to the cardiovascular collapse. The dextro-enantiomer again has the more marked depressive effect on the central nervous system. ${ }^{22}$

The reduced sodium channel affinity and improved potential for safety of levobupivacaine does not seem to occur at the expense of its local anaesthetic action on nerve cells. Indeed, the duration of action for levobupivacaine may be prolonged because of a vasoconstrictor action unique to this isomer. Prolonged nerve blockade was reported as an adverse effect in our study, but levobupivacaine did not appear to be the main culprit. Studies by Aps and Reynolds ${ }^{23}$ on volunteers and by Fernandes and Simmonetti ${ }^{24}$ on isolated human blood vessels have produced contrasting results. Further work is required to clarify this characteristic of levobupivacaine.

The ability to produce purified subgroups of certain drugs has the potential to considerably improve patient safety. Indeed, there may arise an ethical and, subsequently, a legal requirement to prescribe a single drug enantiomer if it has been shown to be the safest preparation, and is equally effective. Our plasma concentration data suggest that bupivacaine toxicity would have been unlikely with the quantities of racemic bupivacaine used, but levobupivacaine would seem to provide an equivalent local anaesthetic agent for peribulbar block.

\section{Acknowledgements}

This study was sponsored by Chiroscience Limited. We are very grateful to all the staff at the Royal Eye Infirmary, Plymouth, particularly Mr NM Evans, Mr VT
Thaller, Mr John Rigby and Mrs Jayne Wootten-Pineles. We also thank Mrs Sue Levie and Mrs Karen Forbes at Inveresk Clinical Research for their assistance in designing the study.

\section{References}

1 Köller C. Ueber die Verwendung des Cocains zur Anasthesitung an Auge. Wien Med Blätter 1884; 7: 1352-1355.

2 Knapp H. On cocaine and its use in ophthalmic and general surgery. Arch Ophthalmol 1884; 13: 402-448.

3 Grizzard WS, Kirk NM, Pavan PR et al. Perforating ocular injuries caused by anesthesia personnel. Ophthalmology 1991; 98: 1011-1016.

4 Fry RA, Henderson J. Local anaesthesia for eye surgery: the periocular technique. Anaesthesia 1989; 45: 14-17.

5 Davis DB. Posterior peribulbar anesthesia. J Cataract Refract Surg 1986; 12: 128-134.

6 Hamilton RC, Gimbel HV, Strunin L. Regional anaesthesia for 12,000 cataract extraction and intraocular lens implantation procedures. Can J Anaesth 1988; 35: 615-623.

7 Davis DB, Mandel MR. Efficacy and complication rate of 16,224 consecutive peribulbar blocks: a prospective multicenter study. J Cataract Refract Surg 1994; 20: 327-337.

8 Shriver PA, Sinha S, Galusha JH. Prospective study of the effectiveness of retrobulbar and peribulbar anaesthesia for anterior segment surgery. J Cataract Refract Surg 1992; 18: 162-165.

9 Albright GA. Cardiac arrest following regional anesthesia with etidocaine or bupivacaine. Anesthesiology 1979; 51: 285-287.

10 Rosenburg PH, Kalso EA, Tuominen MK et al. Acute bupivacaine toxicity as a result of venous leakage under the tourniquet cuff during a Bier's block. Anesthesiology 1983, 58: 95-98.

11 Mazoit JX, Boico O, Samii K. Myocardiac uptake of bupivacaine: pharmacokinetics and pharmacodynamics of bupivacaine enantiomers in the isolated perfused rabbit heart. Anesth Analg 1993; 77: 477-482.

12 Huang YF, Pryor ME, Mather LE et al. Cardiovascular and central nervous system effects of intravenous levobupivacaine and bupivacaine in sheep. Anesth Analg 1998; 86: 797-804.

13 Cox CR, Checketts MR, Mackenzie N et al. Comparison of $\mathrm{S}(-)$-bupivacaine with racemic (RS)-bupivacaine in supraclavicular brachial plexus block. Br J Anaesth 1998; 80 594-598.

14 Miller DK, Haman SM. Graphical aid for determining the power of clinical trials involving two groups. Br J Med 1988; 297: 672-676.

15 House PH, Hollands RH, Schulzer M. Choice of anesthetic agents for peribulbar anesthesia. J Cataract Refract Surg 1991; 17: $80-83$.

16 Morsmann CD, Holden R. The effects of adrenaline, hyaluronidase and age on peribulbar anaesthesia. Eye 1992; 6: 290-292.

17 Döpfmer UR, Maloney DG, Gaynor PA et al. Prilocaine 3\% is superior to a mixture of bupivacaine and lignocaine for peribulbar anaesthesia. Br J Anaesth 1996; 76: 77-80.

18 Aberg G. Toxicological and local anaesthetic effects of optically active isomers of two local anaesthetic compounds. Acta Pharmacol Toxicol 1972; 31: 273-286. 
19 Clarkson CW, Hondeghem LM. Mechanism for bupivacaine depression of cardiac conduction: fast block of sodium channels during the action potential with slow recovery from the block during diastole. Anesthesiology 1985; 62: 396-405.

20 Vanhoutte F, Vereecke J, Verbeke $\mathrm{N}$ et al. Stereoselective effects of the enantiomers of bupivacaine on the electrophysiological properties of the guinea-pig papillary muscle. Br J Pharmacol 1991; 103: 1275-1281.

21 Valenzuela C, Delpon E, Tamkun MM et al. Stereoselective block of human cardiac potassium channels (Kvl.5) by bupivacaine enantiomers. Biophys J 1995; 65: 418-427.
22 Denson DD, Behbehani MM, Gregg RV. Enantiomer-specific effects of an intravenously administered arrythmogenic dose of bupivacaine on neurons of the nucleus tractus solitarius and the cardiovascular system in the anesthetised rat. Reg Anesth 1992; 17: 311-316.

23 Aps C, Reynolds F. An intradermal study of the local anaesthetic and vascular effects of the isomers of bupivacaine. Br J Clin Pharmacol 1978; 6: 63-68.

24 Fernandes L, Simonetti MPB. Vascular effects of S(-)bupivacaine, RS bupivacaine and ropivacaine on isolated strips of human umbilical vein: preliminary results. Reg Anaesth 1997; 22(Suppl 2): 65. 